( С Д.Н. Лаптев, А.О. Емельянов, Е.А. Андрианова, Л.И. Зильберман, И.А. Еремина, Г.Н. Светлова, Т.Л. Титович, О.Б. Безлепкина, В.А. Петеркова

Национальный медицинский исследовательский центр эндокринологии, Москва

ОБОСНОВАНИЕ. В 2018 г. в России появилась система флеш-мониторирования глюкозы (ФМГ) - FreeStyle Libre Flash Glucose Monitoring, которая стала потенциальной альтернативой традиционному самоконтролю глюкозы крови (СМГК). Проведенные к настоящему времени исследования показали преимущества ФМГ по сравнению с СМГК, однако лишь отдельные из них относятся к реальной клинической практике, особенно у детей с СД1.

ЦЕЛЬ. Оценить эффективность ФМГ у детей с сахарным диабетом 1 типа (СД1) в отношении показателей гликемического контроля, возникновения тяжелой гипогликемии и диабетического кетоацидоза, а также удовлетворенность пациентов и их родителей применением ФМГ.

МАТЕРИАЛЫ И МЕТОДЫ. ОДноцентровое проспективное обсервационное когортное исследование. К участию в исследовании были приглашены дети 4-18 лет с СД1 и уровнем гликированного гемоглобина $\left(\mathrm{HbA}_{1 c}\right)$ менее 10,0\% на интенсифицированной инсулинотерапии (путем множественных инъекций - МИИ или непрерывной подкожной инфузии инсулина - НПИИ). Длительность участия пациента в исследовании составляла 6 мес. Исходно и далее каж-

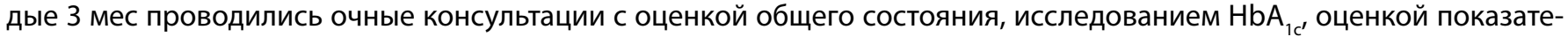
лей гликемии, прогресса в отношении целевых показателей гликемического контроля и коррекцией проводимой терапии. Всего в исследование были включены 228 пациентов (110 мальчиков и 118 девочек), подходящих по критериям включения. Медиана возраста составила 11,2 (8,6-14,7) года, длительность СД1 - 3,8 $(2-7,1)$ года, 136 пациентов получали инсулинотерапию путем НПИИ в течение $1,3(0,8-2,6)$ года.

РЕзУЛЬтАТЫ. В общей группе пациентов, через 3 и 6 мес после начала использования ФМГ, показатели НbА ${ }_{1 c}$ статистически значимо снизились на 0,2\%. Помимо этого, увеличилось количество детей с уровнем $\mathrm{HbA}_{1 c}<7,5 \%$ на 6,1 и 4,9\% на 3-м и 6-м месяце соответственно, однако данные изменения не были статистически значимыми. Число случаев диабетического кетоацидоза при использовании ФМГ снизилось на 74\%, а число случаев тяжелой гипогликемии - на 83\%, таким образом, число эпизодов снизилось в 4 и 6 раз соответственно. Удобство применения и свой опыт использования ФМГ пациенты и/или их родители оценивали по шкале от 0 (полностью согласен) до 4 (совсем не согласен). Большинство детей и родителей положительно (0 или 1) оценили удобство установки и ношения датчика (72,7-98,2\%) и использование системы ФМГ в целом (75,0-96,4\%) по сравнению с СМГК глюкометром (92,3-98,2\%).

ЗАКЛюЧЕНИЕ. Установка и использование ФМГ являются Удобными и комфортными для абсолютного большинства детей и родителей, при этом по сравнению с СМГК применение ФМГ удобнее и проще, а измерение глюкозы гораздо быстрее и менее болезненно.

КЛЮЧЕВЫЕ СЛОВА:СД 1 типа у детей и подростков; системы непрерывного мониторирования глюкозы; НМГ; системы флеш-мониторинга глюкозы; ФМГ; самоконтроль глюкозы крови; СМГК

\title{
THE USE OF FLASH GLUCOSE MONITORING IN CHILDREN WITH TYPE 1 DIABETES MELLITUS IN REAL CLINICAL PRACTICE
}

(c) Dmitry N. Laptev, Andrey O. Emelyanov, Ekaterina A. Andrianova, Lyubov I. Zilberman, Irina A. Eremina, Galina N. Svetlova, Elena V. Titovich, Olga B. Bezlepkina, Valentina A. Peterkova

Endocrinology Research Centre, Moscow, Russia

BACKGROUND: In 2018, a Frestyle Libre flash glucose monitoring system (FGM) appeared in Russia and became a potential alternative to the traditional CGM. Studies carried out to date have shown the advantages of FGM over SMBG, but only a few of them relate to real clinical practice, especially in children with type 1 diabetes.

OBJECTIVE: To evaluate the efficacy of FGM in children with T1DM in relation to glycemic control indicators, the occurrence of severe hypoglycemia and diabetic ketoacidosis, as well as the satisfaction of patients and their parents with the use of FGM.

MATERIALS AND METHODS: Single-center, prospective, observational cohort study. Children 4-18 years old with T1DM and $\mathrm{HbA}_{1 \mathrm{c}}$ level less than $10.0 \%$ were invited to participate in the study on intensified insulin therapy (by MDI or CSII). 
The duration of the patient's participation in the study was 6 months. At baseline and every 3 months thereafter, face-to-face consultations were conducted with an assessment of the general condition, $\mathrm{HbA}_{1 c}$ study, an assessment of glycemic indicators, progress in relation to glycemic control targets and correction of the therapy. A total of 228 patients (110 boys and 118 girls) who met the inclusion criteria were included in the study. The median age was $11.2(8.6-14.7)$ years, the duration of type 1 diabetes was 3.8 (2-7.1), 136 patients received insulin therapy by CSII for $1.3(0.8-2.6)$ years.

RESULTS: In the general group of patients, 3 and 6 months after the start of FGM use, the $\mathrm{HbA}_{1 c}$ values decreased statistically significantly by $0.2 \%$. In addition, the number of children with $\mathrm{HbA}_{1 c}<7.5 \%$ increased by 6.1 and $4.9 \%$ at 3 and 6 months, respectively, but these changes were not statistically significant. The number of cases of DKA when using FGM decreased by $74 \%$, and the number of cases of severe hypoglycemia by $83 \%$, thus the number of episodes decreased by 4 and 6 times, respectively. Patients and / or their parents rated the ease of use and their experience with FGM on a scale from 0 (strongly agree) to 4 (strongly disagree). The majority of children and parents positively ( 0 or 1$)$ assessed the convenience of installing and wearing the sensor (72.7-98.2\%) using the FGM system in general (75.0-96.4\%) and in comparison with the SMBG glucometer (92.3-98.2\%).

CONCLUSION: The installation and use of FGM is convenient and comfortable for the vast majority of children and parents, while compared to SMBG, the use of FGM is more convenient and simpler, and glucose measurement is much faster and less painful.

KEYWORDS: type 1 diabetes mellitus in children and adolescents; T1D; CGM; continuous glucose monitoring; FGM; flash glucose monitoring; $S M B G$; self monitoring of blood glucose

Достижение и поддержание целевых показателей гликемии является одной из ключевых целей лечения сахарного диабета 1 типа (СД1), что необходимо для предупреждения формирования осложнений [1-4]. Регулярный самоконтроль глюкозы крови (СМГК) у детей с СД1 позволяет достигать лучшего уровня гликемического контроля и до недавнего времени был единственным методом измерения глюкозы [5]. При этом СМГК предоставляет лишь частичную информацию о гликемическом профиле, а сама процедура измерения глюкозы болезненна и дискомфортна для пациента, что снижает приверженность пациентов к СМГК.

В 2018 г. в Российской Федерации появилась система флеш-мониторирования глюкозы (ФМГ) — FreeStyle Libre Flash Glucose Monitoring, которая стала потенциальной альтернативой традиционному СМГК, сформировав отдельное направление в непрерывном мониторинге глюкозы (НМГ) - периодически сканируемый НМГ [6]. ФМГ не требует калибровки и предоставляет информацию о текущем уровне глюкозы, тенденции (направления и скорости) изменения глюкозы, график глюкозы за последнее и предыдущее время.

Проведенные к настоящему времени исследования показали преимущества ФМГ по сравнению с СМГК, однако лишь отдельные из них относятся к реальной клинической практике, особенно у детей с СД1.

\section{ЦЕЛЬ}

Оценить эффективность ФМГ у детей с СД1 в отношении показателей гликемического контроля, возникновения тяжелой гипогликемии и диабетического кетоацидоза (ДКА), а также удовлетворенность пациентов и их родителей применением ФМГ.

\section{МАТЕРИАЛЫ И МЕТОДЫ}

\section{Дизайн исследования}

Одноцентровое проспективное обсервационное когортное исследование. К участию в исследовании были приглашены дети в возрасте $>4$ и $<18$ лет с СД1 и уровнем гликированного гемоглобина $\left(\mathrm{HbA}_{1 c}\right)$ менее $10,0 \%$ на интенсифицированной инсулинотерапии (путем множественных инъекций - МИИ или непрерывной подкожной инфузии инсулина - НПИИ).

Процедуры исследования и регистрация показателей

Длительность участия пациента в исследовании составляла 6 мес. Исходно при инициации ФМГ и далее каждые 3 мес проводились очные консультации с оценкой общего состояния, исследованием $\mathrm{HbA}_{1 c^{\prime}}$ оценкой показателей гликемии, прогресса в отношении целевых показателей гликемического контроля и коррекцией проводимой терапии.

Определение уровня $\mathrm{HbA}_{1 с}$ проводилось методом высокоэффективной жидкостной хроматографии на анализаторе BioRad D-10 (BioRad Laboratories, США) в образцах сыворотки крови, взятой утром натощак.

Инициация ФМГ проводилась во время первой очной консультации и включала в себя обучение правилам установки и использования датчика и сканера, принципам измерения глюкозы и анализа данных.

По окончании исследования пациентам или их родителям (в зависимости от возраста ребенка) было предложено заполнить специально разработанные опросники по удобству установки и удовлетворенности использованием ФМГ.

\section{Основной исход исследования}

Изменение $\mathrm{HbA}_{1 c}$ и доля пациентов, достигших $\mathrm{HbA}_{1 c}$ менее 7,5\% к 3-му и 6-му месяцу исследования, по сравнению с исходным уровнем. Изменение частоты случаев ДКА и тяжелой гипогликемии к концу исследования по сравнению с исходным уровнем.

\section{Дополнительные исходы исследования}

Изменение $\mathrm{HbA}_{1 \mathrm{c}}$ в зависимости от его исходного уровня: $<7,5 \%$ и $\geq 7,5 \%$. Удовлетворенность применением ФМГ детей и родителей.

\section{Этическая экспертиза}

Протокол исследования одобрен локальным Комитетом по этике ФГБУ «НМИЦ эндокринологии» Минздрава России, протокол №3 от 14.02.2018. До включения 
Таблица 1. Клинические исходы через 3 и 6 месяцев после инициации флеш-мониторинга глюкозы

\begin{tabular}{|c|c|c|c|c|c|}
\hline Показатель & Исходно & $3 \mathrm{mec}$ & $\mathbf{P}$ & $6 \mathrm{mec}$ & $\mathbf{P}$ \\
\hline $\mathrm{HbA}_{1 c^{\prime}} \%$ & $7,6(6,8-8,9)$ & $7,4(6,8-8,3)$ & $<0,001$ & $7,4(6,8-8,4)$ & 0,042 \\
\hline Пациенты с уровнем <7,5\%, \% & 46,1 & 52,2 & 0,095 & 50 & 0,201 \\
\hline \multicolumn{6}{|l|}{ ДКА } \\
\hline Пациенты с $\geq 1$ эпизодом, \% & 3 & - & - & 0,9 & 0,343 \\
\hline Случаев на 100 пациенто-лет (95\% ДИ) & $7,0(3,0,13,8)$ & - & - & $1,8(0,2,6,3)$ & 0,033 \\
\hline \multicolumn{6}{|l|}{ Тяжелая гипогликемия } \\
\hline Пациенты с $\geq 1$ эпизодом, \% & 4 & - & - & 0,4 & 0,108 \\
\hline Случаев на 100 пациенто-лет (95\% ДИ) & $5,3(1,9,11,5)$ & - & - & $0,9(0,1,4,9)$ & 0,035 \\
\hline
\end{tabular}

Примечание: ДКА - диабетический кетоацидоз

в исследование законные представители пациентов подписали информированное согласие на участие в нем.

\section{Статистический анализ}

Размер выборки предварительно не рассчитывался. Обработка и анализ статистических данных проводились с использованием TIBCO Software Inc. (2017), Statistica (data analysis software system), version 13 и OpenEpi (Open Source Epidemiologic Statistics for Public Health, Atlanta, GA, USA; http://www.openepi.com). Количественные данные представлены в виде медианы и интерквартильного размаха Ме (25-75 перцентиль); качественные данные представлены в виде абсолютных значений (n) и/или частот (\%), данные о частоте эпизодов ДКА и тяжелой гипогликемии представлены в виде частоты эпизодов в пересчете на 100 пациентов в год. Различие между количественными признаками в зависимых выборках оценивалось с помощью Т-критерия Вилкоксона. В случае множественных сравнений использовалась поправка Бонферрони. Различие между качественными признаками оценивалось с помощью точного критерия Фишера, различие между частотой случаев - с помощью точного критерия Mid-P. Значение р менее 0,05 считалось статистически значимым.

\section{РЕЗУЛЬТАТЫ}

Всего в исследование были включены 228 пациентов (110 мальчиков и 118 девочек), подходящих по критериям включения. Медиана возраста составила
11,2 (8,6-14,7) года, длительность СД1 3,8 (2-7,1) года, 136 пациентов получали инсулинотерапию путем НПИИ в течение $1,3(0,8-2,6)$ года.

Гликемический контроль

В общей группе пациентов через 3 и 6 мес после начала использования ФМГ показатели $\mathrm{HbA}_{1 с}$ статистически значимо снизились на 0,2\% (табл. 1). Помимо этого, увеличилось количество детей с уровнем $\mathrm{HbA}_{1 c}<7,5 \%$ на 6, 1 и 4,9\% на 3-м и 6-м месяце соответственно, однако данные изменения не были статистически значимыми.

\section{Диабетический кетоацидоз и тяжелые гипогликемии}

Количество пациентов с $\geq 1$ эпизодом ДКА и тяжелой гипогликемии за 6 мес наблюдения по сравнению с аналогичным периодом до начала использования ФМГ изменилось не значимо (см. табл. 1). В то же время число случаев ДКА при использовании ФМГ снизилось на 74\%, а число случаев тяжелой гипогликемии - на 83\%, таким образом, число эпизодов снизилось в 4 и 6 раз соответственно.

\section{Динамика НbА в в зависимости от его исходного}

уровня

Динамика уровня $\mathrm{HbA}_{1 с}$ также была отдельно проанализирована в двух группах пациентов, имевших исходно разный уровень гликемического контроля: $\mathrm{HbA}_{1 c}<7,5 \%$ $(n=105), \mathrm{HbA}_{1 c} \geq 7,5 \%(n=123)$. У пациентов с исходной компенсацией гликемического контроля ( $\left.\mathrm{HbA}_{1 c}<7,5 \%\right)$ $\mathrm{HbA}_{1 с}$ значимо не изменился ни через 3, ни через 6 мес

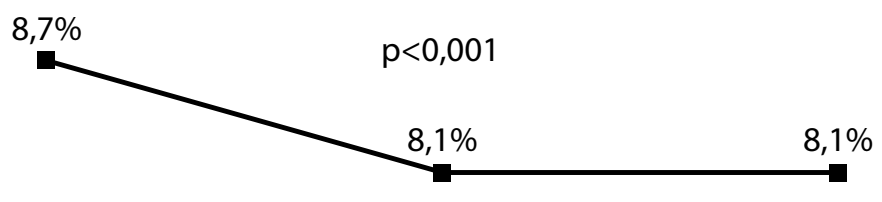

\begin{tabular}{|c|c|c|c|}
\hline & $H / 3$ & & $=-\mathrm{HbA}_{1 \mathrm{c}}<7,5 \%$ \\
\hline $6,7 \%$ & $6,8 \%$ & $6,8 \%$ & \\
\hline
\end{tabular}

\begin{tabular}{lll}
\hline Исходно & $3 \mathrm{mec}$ & $6 \mathrm{mec}$
\end{tabular}

Рисунок 1. Изменение уровня гликированного гемоглобина в зависимости от исходного уровня: $<7,5 \%(n=105), \geq 7,5 \%(n=123)$. 


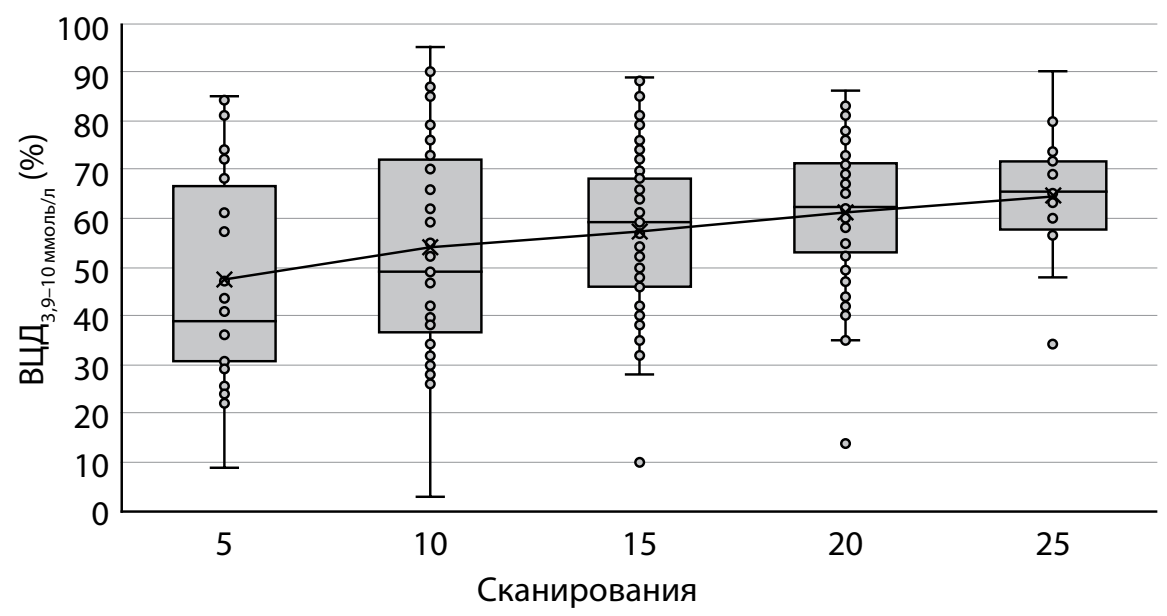

Рисунок 2. Время в целевом диапазоне (ВЦД) 3,9-10 ммоль/л в зависимости от частоты сканирования датчика за сутки.

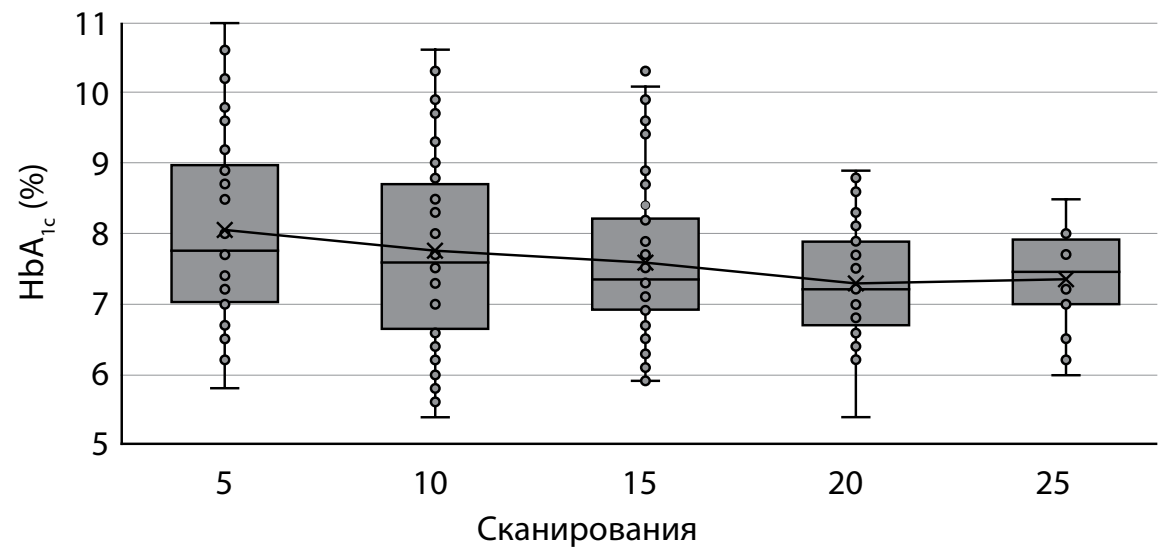

Рисунок 3. Уровень гликированного гемоглобина в зависимости от частоты сканирования датчика за сутки.

использования ФМГ, при этом оставаясь на уровне $<7 \%$ (рис. 1). В свою очередь, у пациентов с декомпенсацией углеводного обмена $\left(\mathrm{HbA}_{1 c} \geq 7,5 \%\right) \mathrm{HbA}_{1 c}$ существенно снизился на 0,6\% к 3-му месяцу наблюдения и сохранился на этом уровне к концу исследования.

\section{Частота сканирования и СМГК}

Во время исследования пациенты сканировали датчик от 5 до 35 раз в течение дня, что в среднем составило 14,9 раза в сутки. Большая частота сканирования была связана с более низкими показателями $\mathrm{HbA}_{1 c^{\prime}}$ а также большим временем в целевом диапазоне 3,9-10,0 ммоль/л, при этом такая закономерность сохранялась при частоте сканирований более 15 раз в сутки (рис. 2, 3).

После инициации ФМГ средняя частота СМГК с помощью глюкометра значимо снизилась с 8,6 до 1,8 измерения гликемии в сутки ( $<<0,001)$. Таким образом, на фоне использования ФМГ потребность в СМГК с помощью глюкометров снизилась в 4,9 раза.

\section{Оценка удовлетворенности использования ФМГ}

Удобство применения и свой опыт использования ФМГ пациенты и/или их родители оценивали по шкале от 0 (полностью согласен) до 4 (совсем не согласен) (рис. 4). Большинство детей и родителей положительно (0 или 1) оценили удобство установки и ношения датчика (72,7-98,2\%) и использование системы ФМГ в целом (75,0-96,4\%) по сравнению с СМГК глюкометром (92,3-98,2\%).

\section{ОБСУЖДЕНИЕ}

Данное исследование демонстрирует, что у детей с СД1 в возрасте от 4 до 18 лет использование ФМГ, по сравнению с СМГК, позволяет улучшить показатели $\mathrm{HbA}_{1 c^{\prime}}$ в том числе в подгруппе пациентов с недостаточным уровнем гликемического контроля, уменьшить число эпизодов тяжелой гипогликемии и ДКА и сократить частоту СМГК, повысить приверженность пациентов к самоконтролю глюкозы, являясь при этом удобным в использовании инструментом контроля глюкозы. В целом эти результаты отражают данные, полученные в других клинических исследованиях и сложившуюся практику клинического применения ФМГ.

В проспективном наблюдательном исследовании SELFY у детей в возрасте 4-17 лет $(\mathrm{n}=76)$ с СД1 через 8 нед от начала использования ФМГ отмечалось снижение $\mathrm{HbA}_{1 c}$ на 0,4\% исходного уровня (p<0,0001), время в целевом диапазоне увеличилось на 0,9 ч в сутки ( $p=0,005)$, частота СМГК при помощи глюкометра сокра-

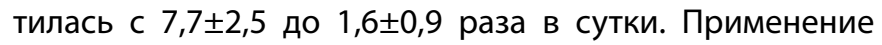
ФМГ ассоциировалось с улучшением показателей общей удовлетворенности терапией при оценке по опроснику The Diabetes Treatment Satisfaction Questionnaire (DTSQ) в сравнении с исходными показателями ( $<<0,0001)$ [7].

По данным проспективного наблюдательного исследования, проведенного А. Messaaoui и соавт. [8], применение ФМГ у детей с СД1 в возрасте 4-20 лет $(n=334)$ 
Установка/ношение ФМГ

Дети

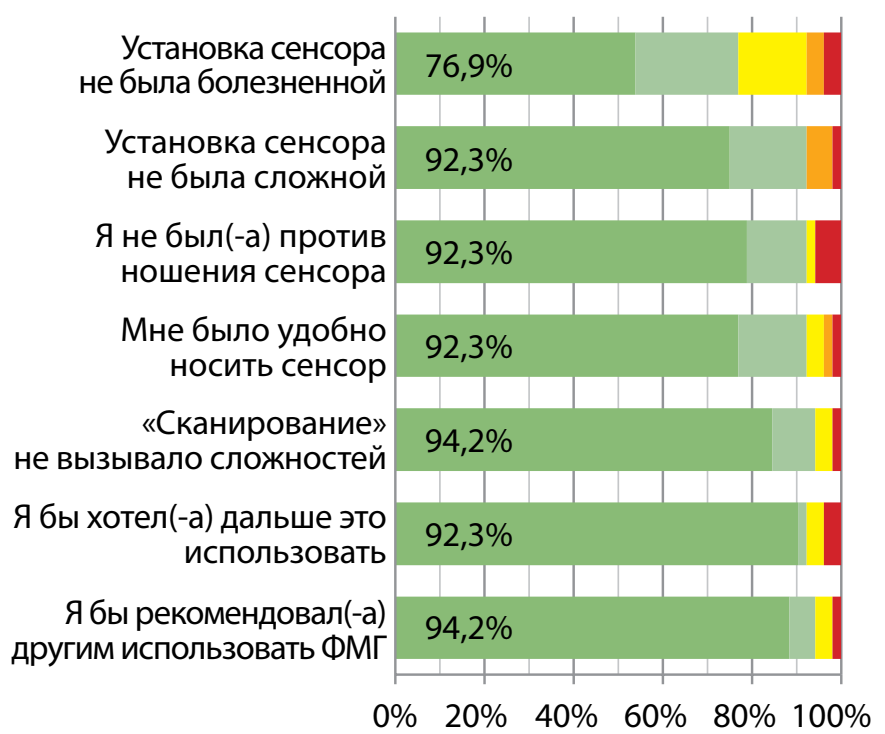

\section{Использование ФМГ}

Дети

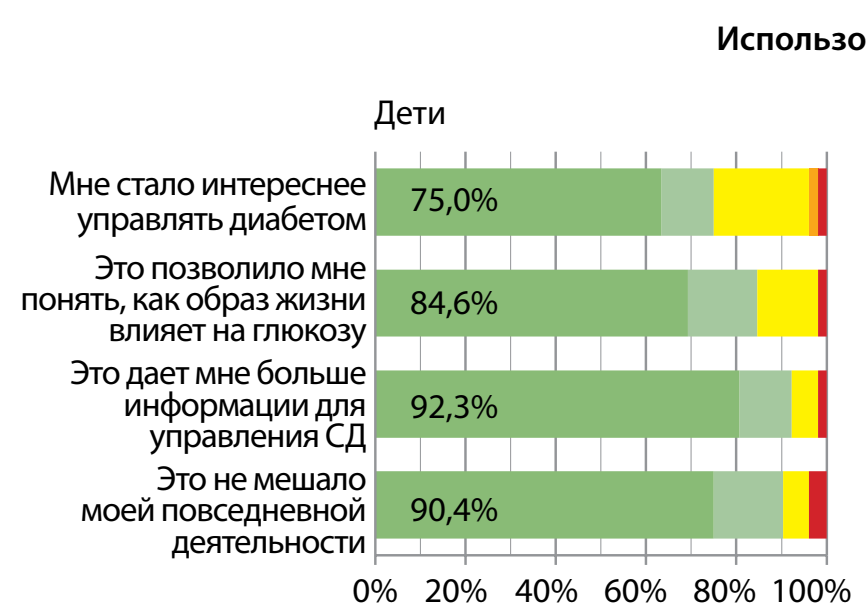

Родители

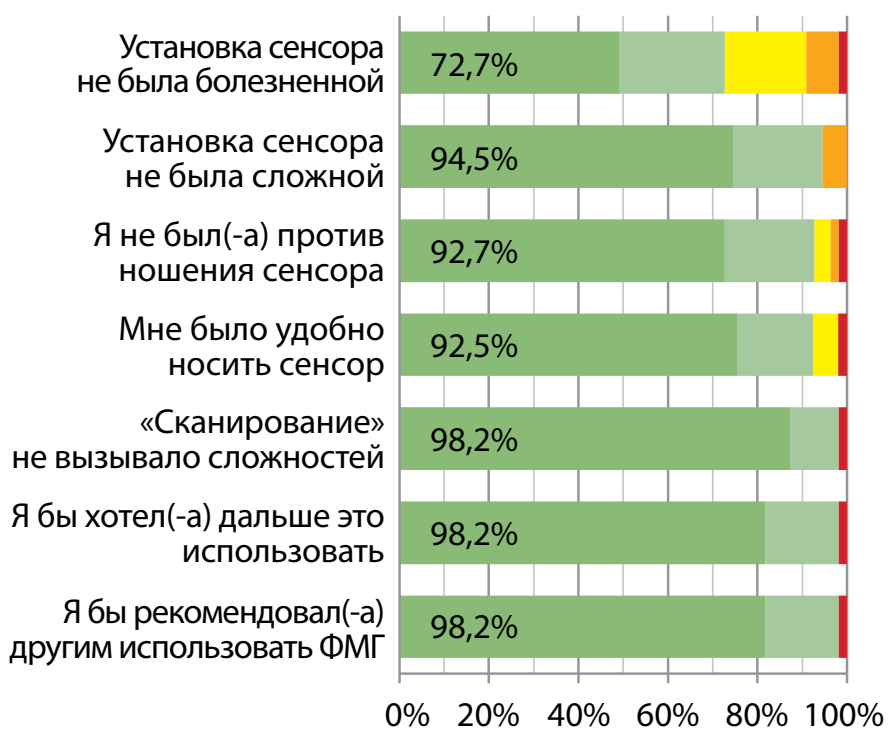

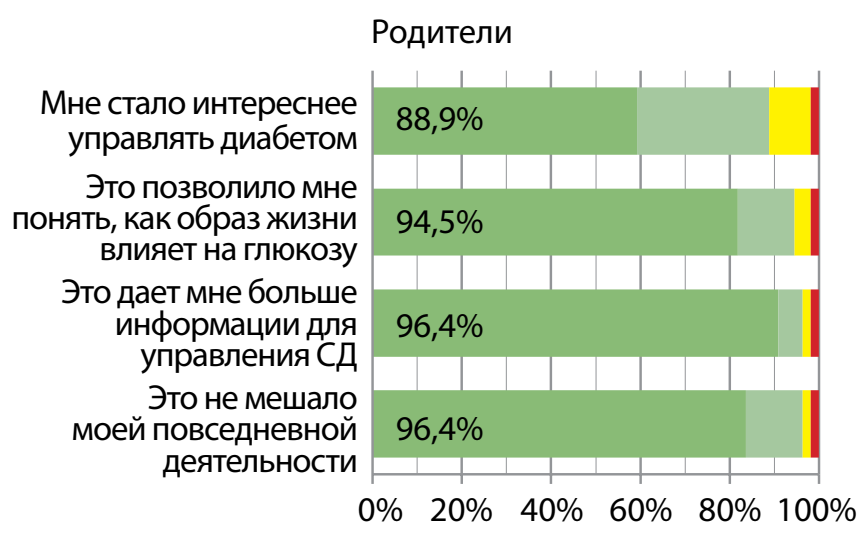

\section{Использование ФМГ по сравнению с глюкометром}

Дети

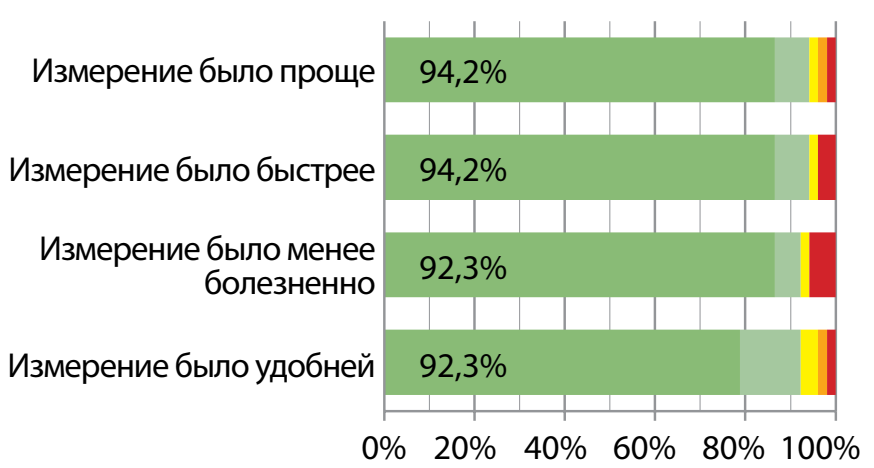

Родители

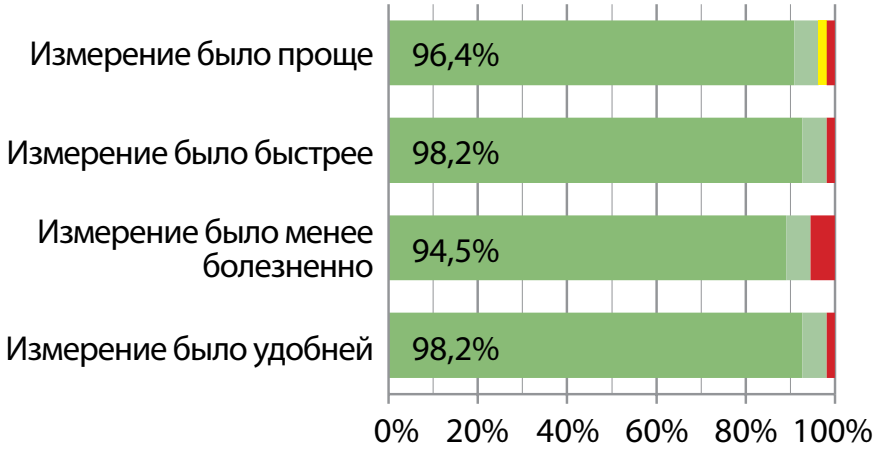

\footnotetext{
- Полностью согласен

Согласен

- Нейтрален

- Не согласен

- Совсем не согласен
} 
в условиях реальной практики в течение 12 мес позволяет уменьшить число эпизодов тяжелой гипогликемии на 53\% В сравнении с СМГК ( $p=0,012)$. В другом проспективном наблюдательном исследовании А. Hayek и соавт. [9] у детей и молодых людей 13-19 лет с СД1 (n=47) применение ФМГ в течение 3 мес сопровождалось уменьшением тревожности, связанной со страхом развития гипогликемии $(p=0,0001)$, повышением качества жизни $(p=0,002)$, снижением уровня $\mathrm{HbA}_{1 c}$ в среднем на $0,66 \%(p=0,008)$, а также уменьшением частоты возникновения эпизодов гипогликемии $(p=0,023)$ от исходных значений.

В наблюдательном исследовании, проведенном в Испании у детей 4-18 лет $(n=145)$ с СД1, отмечалось снижение $\mathrm{HbA}_{1 c}$ на 1,96\% $(\mathrm{p}=0,04)$ в группе пациентов с недостаточным уровнем гликемического контроля $\left(\mathrm{HbA}_{1 c}\right.$ 9,7\%) через 3 мес от начала применения ФМГ. Общая частота эпизодов тяжелой гипогликемии сократилась с 4,2 до 0,2 на 100 пациентов в год через 12 мес от начала применения ФМГ. При этом наименьшая частота легких гипогликемий отмечалась у пациентов с частотой ежедневных сканирований $>10$ раз $(p=0,05)[10]$.

В исследовании J. Suzuki и соавт. [11] у 85 детей с СД1 была выявлена положительная корреляция частоты сканирований с продолжительностью времени в целевом диапазоне $(r=0,719 ; P<0,0001)$ и обратная корреляция с временем выше диапазона ( $r=-0,743 ; P<0,0001)$, средним уровнем глюкозы и уровнем $\mathrm{HbA}_{1 c}$ и расчетным значением $\mathrm{HbA}_{1 c}(r=-0,765,-0,815,-0,793$ соответственно, $\mathrm{p}<0,0001)$.

Применение ФМГ позволяет пациентам и родителям лучше выявлять гипогликемии, в том числе возникающие в ночной период, а также повышать приверженность к регулярному измерению уровня глюкозы. В проспективном наблюдательном исследовании A. Deeb и соавт. [12] у детей (средний возраст 11,2 года) с СД1 $(\mathrm{n}=75)$ при использовании ФМГ эпизоды ночной гипогликемии были выявлены у 90\% пациентов, в то время как при использовании глюкометров - только у 16,6\% пациентов ( $<<0,001)$. Во время применения ФМГ отмечалось увеличение частоты самоконтроля глюкозы пациентами: средняя частота сканирований составила 11,6 раза в сутки по сравнению с 2,87 раза в сутки ( $<<0,001)$ при использовании глюкометров.

Дети с СД1, применявшие ФМГ ( $\mathrm{n}=347)$, отмечают, что использование данной технологии позволяет не только избегать проколов пальцев (85,9\% пациентов), но и получать больше информации об уровне глюкозы, в частности, в ночной период (60,4\% пациентов), легче изменять свои привычки и корректировать образ жизни (89,5\% пациентов), чаще определять свой уровень глюкозы (70,6\% пациентов) [13].

\section{ЗАКЛЮЧЕНИЕ}

Применение ФМГ у детей в возрасте от 4 до 18 лет с СД1 имеет следующие клинические преимущества по сравнению с СМГК:

- улучшение показателей $\mathrm{HbA}_{1 c}$ на 0,2\% в общей группе пациентов;

- более выраженное снижение $\mathrm{HbA}_{1 с}$ на 0,6\% в группе пациентов с недостаточным уровнем гликемического контроля $\left(\mathrm{HbA}_{1 \mathrm{c}}>7,5 \%\right)$;

- снижение риска тяжелой гипогликемии на 83\% и ДКА на 74\%;

- уменьшение потребности в инвазивном СМГК в 5 раз и повышение приверженности пациентов к измерению глюкозы в 1,7 раза до 15 сканирований в день.

Эффективность использования ФМГ во многом зависит от интенсивности и времени использования системы. Большая частота сканирования способствует увеличению времени в целевом диапазоне и снижению уровня $\mathrm{HbA}_{1 c^{\circ}}$

Установка и использование ФМГ являются удобными и комфортными для абсолютного большинства детей и родителей, при этом по сравнению с СМГК применение ФМГ удобнее и проще, а измерение глюкозы гораздо быстрее и менее болезненно.

\section{ДОПОЛНИТЕЛЬНАЯ ИНФОРМАЦИЯ}

Финансирование проекта. Работа выполнена в рамках клинической апробации «Оказание специализированной медицинской помощи детям и подросткам с сахарным диабетом 1 типа с использованием системы Flash-мониторинга глюкозы».

Конфликт интересов. Авторы декларируют отсутствие явных и потенциальных конфликтов интересов, связанных с публикацией настоящей статьи.

Участие авторов. Петеркова В.А. - научное руководство, дизайн и планирование исследования; Лаптев Д.Н. - выгрузка данных из регистра, анализ и статистическая обработка полученных данных, написание текста; Емельянов А.О. - ведение регистра пациентов, выгрузка данных из регистра, написание и редактирование текста; Андрианова Е.А. - ведение регистра пациентов, выгрузка данных из регистра; Зильберман Л.И. - ведение регистра пациентов, выгрузка данных из регистра; Еремина И.А. - ведение регистра пациентов, выгрузка данных из регистра; Светлова Г.Н. - ведение регистра пациентов, выгрузка данных из регистра; Титович Е.В. - ведение регистра пациентов, выгрузка данных из регистра; Безлепкина О.Б. - научное руководство, дизайн и планирование исследования. Все авторы одобрили финальную версию статьи перед публикацией, выразили согласие нести ответственность за все аспекты работы, подразумевающую надлежащее изучение и решение вопросов, связанных с точностью или добросовестностью любой части работы.

\section{СПИСОК ЛИТЕРАТУРЫ | REFERENCES}

1. Дедов И.И., Шестакова М.В., Майоров А.Ю., и др. Алгоритмы специализированной медицинской помощи больным сахарным диабетом. Под редакцией И.И. Дедова, М.В. Шестаковой, А.Ю. Майорова 9-й выпуск // Сахарный диабет. - 2019. - Т. 22. — №1S1. - C. 1-144 [Dedov II, Shestakova MV, Mayorov AYu, et al. Standards of specialized diabetes care. Ed. by II Dedov, MV Shestakova, AY Mayorov. Diabetes Mellitus. 2019;22(S1):1-144 (In Russ.)]. doi: https://doi.org/10.14341/DM221S1
2. Петеркова В.А., Шестакова М.В., Безлепкина О.Б., и др. Сахарный диабет 1 типа у детей // Сахарный диабет. - 2020. - Т. 23. №1S. - C. 4-40. [Peterkova VA, Shestakova MV, Bezlepkina OB, et al. Diabetes mellitus type 1 in childhood. Diabetes mellitus. 2020;23(1S):4-40. (In Russ.)]. doi: https://doi.org/10.14341/DM12504

3. Дедов И.И., Шестакова М.В., Майоров А.Ю. Сахарный диабет 1 типа у взрослых // Сахарный диабет. — 2020. - Т. 23. — № 1S. C. 42-1 14. [Dedov II, Shestakova MV, Mayorov AY, et al. Diabetes mellitus type 1 in adults. Diabetes mellitus. 2020;23(15):42-114. (In Russ.)]. doi: https://doi.org/10.14341/DM12505 
4. DiMeglio LA, Acerini CL, Codner E, et al. ISPAD Clinical Practice Consensus Guidelines 2018: Glycemic control targets and glucose monitoring for children, adolescents, and young adults with diabetes. Pediatr Diabetes. 2018;19:105-114. doi: https://doi.org/10.1111/pedi.12737

5. Miller KM, Beck RW, Bergenstal RM, et al. Evidence of a Strong Association Between Frequency of Self-Monitoring of Blood Glucose and Hemoglobin A1c Levels in T1D Exchange Clinic Registry Participants. Diabetes Care. 2013;36(7):2009-2014. doi: https://doi.org/10.2337/dc12-1770

6. Danne T, Nimri R, Battelino T, et al. International Consensus on Use of Continuous Glucose Monitoring. Diabetes Care. 2017:40(12):1631-1640. doi: https://doi.org/10.2337/dc17-1600

7. Campbell FM, Murphy NP, Stewart C, et al. Outcomes of using flash glucose monitoring technology by children and young people with type 1 diabetes in a single arm study. Pediatr Diabetes. 2018;19(7):1294-1301. doi: https://doi.org/10.1111/pedi.12735

8. Messaaoui A, Tenoutasse S, Crenier L. Flash Glucose Monitoring Accepted in Daily Life of Children and Adolescents with Type 1 Diabetes and Reduction of Severe Hypoglycemia in Real-Life Use. Diabetes Technol Ther. 2019;21(6):329-335. doi: https://doi.org/10.1089/dia.2018.0339
9. Al Hayek AA, Robert AA, Al Dawish MA. Evaluation of FreeStyle Libre Flash Glucose Monitoring System on Glycemic Control, Health-Related Quality of Life, and Fear of Hypoglycemia in Patients with Type 1 Diabetes. Clin Med Insights Endocrinol Diabetes. 2017;10:117955141774695. doi: https://doi.org/10.1177/1179551417746957

10. Leiva-Gea I, Vázquez JG, Jurado FRL, et al. Introduction of flash glucose monitoring in children with Type 1 diabetes: experience of a single-centre in Spain. ESPE Abstracts. 2019;92:LB-20.

11. Suzuki J, Urakami T, Yoshida K, et al. Association between scanning frequency of flash glucose monitoring and continuous glucose monitoring-derived glycemic makers in children and adolescents with type 1 diabetes. Pediatr Int. 2021;63(2):154-159. doi: https://doi.org/10.1111/ped.14412

12. Deeb A, Yousef H, Al Qahtani N, et al. Novel ambulatory glucose-sensing technology improves hypoglycemia detection and patient monitoring adherence in children and adolescents with type 1 diabetes. J Diabetes Metab Disord. 2019;18(1):1-6. doi: https://doi.org/10.1007/s40200-018-0351-9

13. Vergier J, Samper M, Dalla-Vale F, et al. Evaluation of flash glucose monitoring after long-term use: A pediatric survey. Prim Care Diabetes. 2019;13(1):63-70. doi: https://doi.org/10.1016/j.pcd.2018.08.004

\section{ИНФОРМАЦИЯ ОБ АВТОРАХ [AUTHORS INFO]}

*Емельянов Андрей Олегович, к.м.н. [Andrey O. Emelyanov, MD, PhD]; адрес: Россия, 117036, г. Москва, ул. Дм. Ульянова, д. 11 [address: 11 Dm. Ulyanova str., 117036, Moscow, Russia], ORCID: https://orcid.org/0000-0002-3742-0231; eLibrary SPIN: 8110-5540; e-mail: endiab@mail.ru

Лаптев Дмитрий Никитич, д.м.н. [Dmitry N. Laptev, MD, PhD]; ORCID: https://orcid.org/0000-0002-4316-8546; eLibrary SPIN: 2419-4019; e-mail: laptevdn@ya.ru

Андрианова Екатерина Андреевна, К.M.H. [Ekaterina A. Andrianova, MD, PhD]; eLibrary SPIN: 7496-4580;

e-mail: katandr13@list.ru

Зильберман Любовь Иосифовна, к.м.н. [Lubov I. Zilberman, MD, PhD]; ORCID: https://orcid.org/0000-0002-0316-8314; eLibrary SPIN: 4488-7724; e-mail: zilbermanl@yandex.ru

Еремина Ирина Александровна, к.M.н. [Irina A. Eremina, MD, PhD]; ORCID: https://orcid.org/0000-0003-2333-3935; eLibrary SPIN: 9411-4710; e-mail: ieremina58@gmail.com

Светлова Галина Николаевна, к.M.H. [Galina N. Svetlova, MD, PhD]; ORCID: https://orcid.org/0000-0002-4328-2090; eLibrary SPIN: 9356-2673; e-mail: g_svetlova@mail.ru

Титович Елена Витальевна, к.м.Н., в.н.с. [Elena V. Titovich, MD, PhD, leading research associate];

ORCID: https://orcid.org/0000-0001-7821-3979; eLibrary SPIN: 7994-0797; e-mail: lenatitovich@mail.ru

Безлепкина Ольга Борисовна, А.м.н. [Olga B. Bezlepkina, MD, PhD]; ORCID: https://orcid.org/0000-0001-9621-5732; eLibrary SPIN: 3884-0945; e-mail: olgabezlepkina@mail.ru

Петеркова Валентина Александровна, д.м.н., профессор, академик PAH [Valentina A. Peterkova, MD, PhD,

Professor, academician of Russian Academy of Medical Sciences]; ORCID: https://orcid.org/0000-0002-5507-4627;

eLibrary SPIN: 4009-2463; e-mail: peterkovava@hotmail.com

\section{ЦИТИРОВАТЬ:}

Лаптев Д.Н., Емельянов А.О., Андрианова Е.А., Зильберман Л.И., Еремина И.А., Светлова Г.Н., Титович Е.В., Безлепкина О.Б., Петеркова В.А. Применение Flash-мониторинга глюкозы у детей с сахарным диабетом 1 типа в реальной клинической практике // Сахарный диабет. — 2021. - Т. 24. — №6. - С. 504-510. doi: https://doi.org/10.14341/DM12817

\section{TO CITE THIS ARTICLE:}

Laptev DN, Emelyanov AO, Andrianova EA, Zilberman LI, Eremina IA, Svetlova GN, Titovich EV, Bezlepkina OB, Peterkova VA. The use of Flash glucose monitoring in children with type 1 diabetes mellitus in real clinical practice. Diabetes Mellitus. 2021;24(6):504-510. doi: https://doi.org/10.14341/DM12817 